nutrition while one patient required palliative bowel decompression. Tube placement was successful in all patients. In 23 patients, PTEG was performed by approaching the left side of the neck. One patient required a right side approach. A specialized 15 Fr size tube (45 cm in length) was used during initial placement. The mean postoperative length of stay was 18.9 (6 - 48) days. Postoperative adverse events included accidental tube dislodgement in 3 patients, thyroid gland mispuncture in 2 patients, left inferior thyroid artery injury in one patient and tracheoesophageal fistula in one patient. There was no procedure-related mortality and only one patient died within 30 days after the procedure due to underlying comorbidities. Ten patients were discharged with some oral intake and 2 patients recovered full oral intake, enabling removal of the tube.

Conclusions PTEG is feasible in patients requiring long term gut access but is not suitable candidates for PEG. It is a relatively safe procedure and should be offered as a more comfortable alternative to nasogastric tubing.

\section{IDDF2019-ABS-0061 TECHNICAL FEASIBILITY OF A NEWLY DESIGNED BENDABLE FORCEPS FOR DIFFICULT ENDOSCOPIC TISSUE SAMPLINGS}

'Dong Seok Lee*, 'Ji Won Kim, ${ }^{1}$ Kook Lae Lee, ${ }^{1}$ Byeong Gwan Kim, ${ }^{1}$ Yong Jin Jeong, ${ }^{1}$ Su Hwan Kim, ${ }^{2}$ Jeong-Sik Byeon. 'Seoul National University Boramae Medical Center, Korea, South; ${ }^{2}$ Asan Medical Center, University of Ulsan College of Medicine, Korea, South

\subsection{6/gutjnl-2019-IDDFabstracts. 133}

Background Biopsies with conventional forceps beyond the operating range are difficult and cumbersome. Thus, we developed a new forceps and evaluated its technical feasibility.

Methods A bendable forceps was constructed with two channels and a two-stage knob. The bending motion-related structures were designed to improve the range of motion(figure 1). For performance comparisons, we established a scoring system for the evaluation of the forceps, with $\underline{9}$ special cases. All procedures were performed by 5 expert endoscopists.

Results Biopsies with the bendable forceps were easy and faster than those performed with conventional forceps in the 9 cases. The mean evaluation scores were $6.2 \pm 0.4$ and $1.96 \pm 0.2$ for the bendable and conventional forceps, respectively $(P<0.001)$.
Conclusions This preliminary study showed that the newly developed bendable forceps allowed for a wide range of motion and is more effective than conventional forceps for endoscopic biopsies in complex GI lesions.

\section{IDDF2019-ABS-0062 GASTRIC HYPERPLASTIC POLYPS ARE ASSOCIATED WITH AN INCREASED RISK OF COLORECTAL NEOPLASIA IN THE CHINESE POPULATION}

${ }^{1}$ Xiaoyong Wang*, ${ }^{1}$ Ruan-ping Zhou, ${ }^{2}$ Jia Wu. 'Department of Gastroenterology, Changzhou No. 2 People's Hospital, Affiliated with Nanjing Medical University, China; 'Department of Gynecology, Changzhou No. 2 People's Hospital, Affiliated with Nanjing Medical University, China

\subsection{6/gutjnl-2019-IDDFabstracts. 134}

Background There are rare studies which evaluated the relationship between gastric hyperplastic polyps and synchronous colorectal neoplasia. To address this issue, we performed this retrospective, case-control study.

Methods Patients with sporadic gastric hyperplastic polyps who underwent colonoscopy simultaneously were consecutively enrolled. Each patient was compared with two randomly selected age and sex-matched controls without gastric polyps who also underwent colonoscopy in the same period in Changzhou No.2 people's hospital between January 2015 and December 2017. The urease breath test or rapid urease test was used to determine $H$. pylori. Data of patient's demographics and characteristics of the gastrointestinal polyps were collected.

Results This study involved 106 patients with gastric hyperplastic polyps and 212 age and sex-matched controls without gastric polyps among 4200 patients who underwent gastroscopy and colonoscopy simultaneously. There was no significant difference in tobacco smoking, alcohol drinking, Diabetes, H. pylori infection and blood lipids between the two groups $(P>0.05)$. In the gastric hyperplastic polyp group, the prevalence of colorectal neoplasia which included adenoma and cancer was 29.2\%; however, in the controls, the prevalence of colorectal neoplasia was $18.4 \%$. There was a significantly higher rate of colorectal neoplasia $(\mathrm{OR}=1.83,95 \% \mathrm{CI}=$ $1.06-3.16)$ in the gastric hyperplastic polyp group than in the control group. There was no significant difference in the size, number and distribution of colorectal neoplasia between the
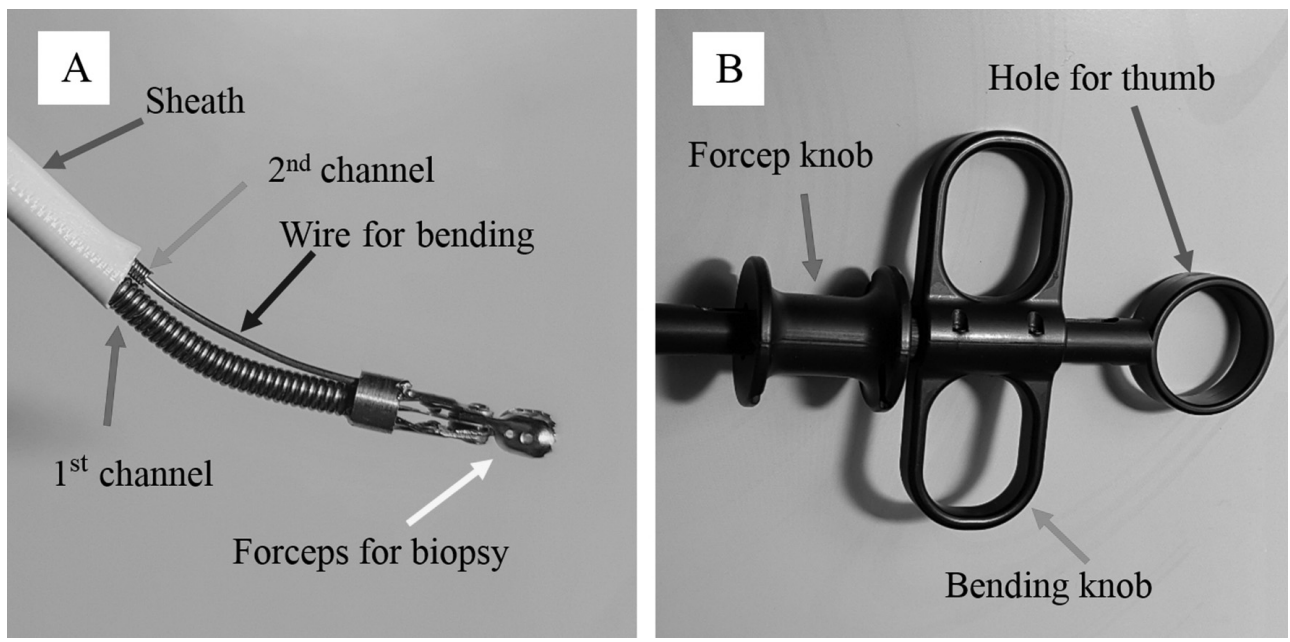